\title{
Room temperature absorption in laterally biased quantum infrared detectors fabricated by MBE regrowth
}

\author{
Álvaro Guzmán*, Rocío San-Román, Adrián Hierro \\ Instituto de Sistemas Optoelectrónicos y Microtecnología (ISOM), ETSI Telecomunicación, Ciudad Universitaria s/n, 28040 Madrid, Spain
}

\begin{abstract}
A B S T R A C T
In this paper, we show room temperature operation of a quantum well infrared photodetector (QWIP) using lateral conduction through ohmic contacts deposited at both sides of two n-doped quantum wells. To reduce the dark current due to direct conduction in the wells, we apply an electric field between the quantum wells and two pinch-off Schottky gates, in a fashion similar to a field effect device. Since the normal incidence absorption is strongly reduced in intersubband transitions in quantum wells, we first analyze the response of a detector based on quantum dots (QD). This QD device shows photocurrent signal up to $150 \mathrm{~K}$ when it is processed in conventional vertical detector. However, it is possible to observe room temperature signal when it is processed in a lateral structure. Finally, the room temperature photoresponse of the QWIP is demonstrated, and compared with theory. An excellent agreement between the estimated and measured characteristics of the device is found.
\end{abstract}

Keywords:

A1. Nanostructures

A3. Molecular beam epitaxy

A3. Quantum wells

B1. Arsenides

B2. Semiconducting III-V materials

B3. Infrared devices

\section{Introduction}

Quantum well infrared photodetectors based on GaAs have been chosen as good candidates to develop high yield IR cameras. The GaAs/AlGaAs material system allows the quantum well shape to be tailored over a range wide enough to enable light detection at wavelengths longer than $3.5 \mu \mathrm{m}$. Easy growth and process of these materials, makes it possible to obtain large uniform focal plane arrays of QWIP's tuned to detect light at wavelengths from $3.5 \mu \mathrm{m}$ to more than $25 \mu \mathrm{m}$. Moreover, hybrid devices combining a focal plane array with a read out integrated circuit made on $\mathrm{Si}$, bonded together by flip-chip techniques have been developed, and in fact, they are already commercially available $[1,2]$.

However, infrared cameras based on QWIPs still show important drawbacks. For example, the need of lowering the operating temperature to cryogenic regimes to reduce the dark current, or the strong reduction in the light absorption in normal incidence conditions due to the selection rules of intraband transitions. The latter condition, normally obliges to etch diffraction gratings on the active surface of the pixels to scatter the light $[3,4]$. As a consequence of all these drawbacks, the QWIP based cameras are generally mounted inside expensive and heavy cryostats, which make the whole system very difficult to be carried by an operator, restricting its use to surveillance or security systems.

Therefore, one of the main objectives is to develop quantum IR detectors, which can operate at room temperature in normal incidence conditions. Some authors have proposed the use of quantum dots in the active region of the detector [5]. The zerodimensional nature of their bound energy states is expected to inhibit phonon scattering, thus increasing the relaxation times, and consequently, more efficient detection is predicted, since photoexcited carriers are less likely to be captured in the quantum dots before being swept away as a photocurrent, leading to increased extraction efficiency and increased operating temperatures. Besides, the normal incidence restrictions imposed in the case of the quantum wells are relaxed in QD structures. In those cases, thick AlGaAs layers are normally grown at both sides of the structure to further reduce the dark current. With these considerations, it is possible to find some works reporting single QDIP devices working at room temperature [6,7].

On the other hand, Chu et al. reported lateral intersubband photocurrent on self-assembled InAs/GaAs QD at normal incidence at low temperature. In this lateral configuration, the contacts are deposited at both sides of the quantum well, parallel to the surface. Thus, the confined charge carriers can be excited into the twodimensional wetting layer (WL) and the carrier transport can take place within the WL in an in-plane electric field. [8,9].

In 2007, Alsing et al. [10] proposed a novel quantum IR detector structure with a lateral biasing scheme, which can in theory operate at higher temperatures. They considered a double quantum well (QW) structure (Fig. 1(a)) consisting of an n-type doped QW tunnel-coupled with another n-type doped QW. Both wells are laterally connected by means of two ohmic junctions (collector contacts) at the sides of the device. Furthermore, two pinch-off Schottky gates are deposited to reduce the direct conduction between the lateral contacts through the QW. This conduction is 
reduced by applying an electric field to the pinch-off gate in a fashion similar to a field effect transistor at each of the wells. When the electrons in one of the wells are promoted from the ground state to the excited state (Fig. 1(b)), they tunnel out to the other

a

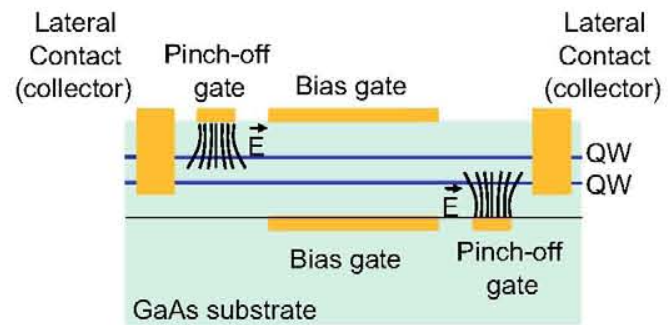

b

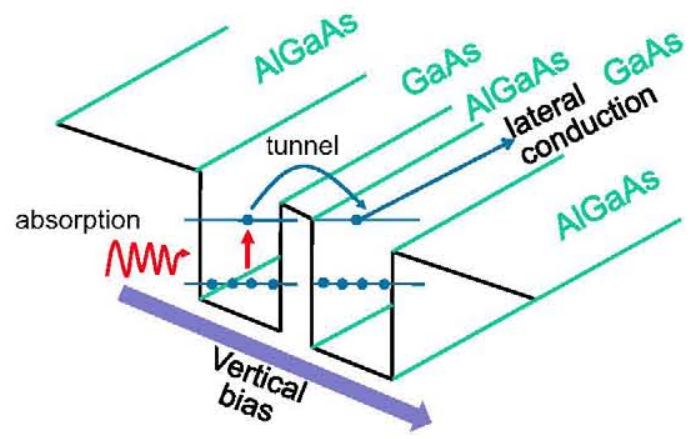

Fig. 1. Cross-section of the structure (a) and band profile (b) of a laterally biased photodetector. The lateral contacts are used to collect the photocurrent. The pinchoff gates allow to create an electric field to reduce the dark current. The bias gates can be employed to bend the bands, tuning the absorption wavelength of the device. The band profile summarizes the work principles of this detector. well. The electrons are then swept to the collector region by means of the lateral bias. It is also possible to apply a vertical bias (parallel to the growth direction) using the bias gate. In this case, there is a shift in the relative energy between the levels of the first well, which allows the tuning of the peak wavelength response of the detector. As a general advantage, since the dark current of the device can be reduced by means of the control gate, it is expected to obtain IR detection at room temperature.

In this work, we report the fabrication of the lateral structure using MBE regrowth of the active region on top of the ion implanted surfaces. We first assess the viability of the technology on a Quantum Dot IR detector, comparing the response of such a device processed into two different structures: conventional vertical detector and laterally biased detector. As a result, we obtained room temperature operation laterally biased, while $150 \mathrm{~K}$ is the highest operation temperature we could measure for conventional processing.

The quantum well device was processed into laterally biased configuration. It was tested at room temperature, and compared with theory in several aspects: $I-V$ characteristics and position of the absorption peak. We achieved photoresponse at room temperature at $8.5 \mu \mathrm{m}$, obtaining excellent agreement between experiments and theory in all cases.

\section{Growth and processing}

The processing steps of the sample are detailed in Fig. 2. The $\mathrm{AlGaAs} / \mathrm{GaAs}$ quantum wells were grown by molecular beam epitaxy using a RIBER 32 system. One of the major difficulties of this structure is the presence of the bias and pinch-off gates at the bottom side of the device. They have to be deposited before the growth of the epilayer. In our case, those gates were fabricated by selective ion implantation on a 2" GaAs undoped wafer. a

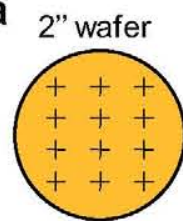

Alignment marks Etching (RIE)

b

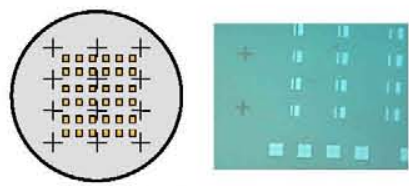

Windows for

ion implantation

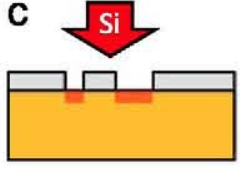

Ion implantation of Si atoms

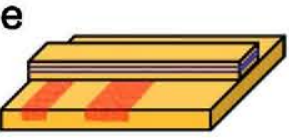

Mesa etching $f$

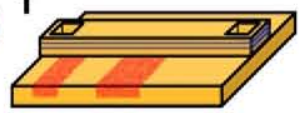

Etching of

lateral contacts

d
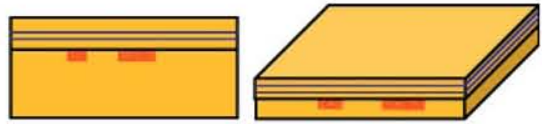

Regrowth of the structure by MBE

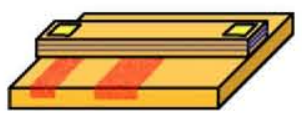

Filling with AuGe/Au

$\mathrm{g}$

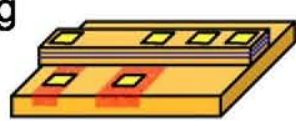

Metallization of Schottky Bias and pinch-off gates

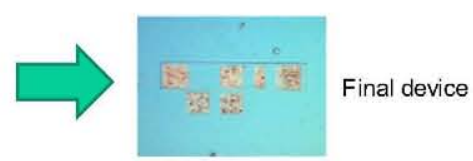

Fig. 2. Processing steps in the fabrication of the laterally biased device shown in Fig. 1. 
In the first step (a), we etched several alignment marks by reactive ion etching (RIE) in order to further align the rest of the processing steps. Then, a series of windows were open in a photoresist (b) and the whole wafer was exposed to the $\mathrm{Si}^{+}$ion beam using a dose of $4 \times 10^{13} \mathrm{~cm}^{-2}$ and an implantation energy of $180 \mathrm{keV}$ (c). The peak of the vertical implantation profile $\left(2 \times 10^{18} \mathrm{~cm}^{-3}\right)$ was designed to be $500 \mathrm{~nm}$ deep from the surface.

After the implantation, the sample was immersed in a solution of $\mathrm{H}_{2} \mathrm{SO}_{4}: \mathrm{H}_{2} \mathrm{O}_{2}: \mathrm{H}_{2} \mathrm{O}$ 1:8:80 for 2 min to clean and etch the surface and was then rinsed with DI water. It was further left in flowing DI water for $15 \mathrm{~min}$ to deposit a protective oxide layer prior to its loading it into the MBE system.

The epilayer was grown at $600{ }^{\circ} \mathrm{C}$ with a V/III beam equivalent pressure ratio of 25 (d). The growth was monitored by in situ reflection high energy electron diffraction. After the oxide desorption at $580^{\circ} \mathrm{C}$ we raised the temperature till the transition between the $(2 \times 4)-\alpha$ and the $(2 \times 4)-\beta$ surface reconstructions was observed at $600^{\circ} \mathrm{C}$ [11]. The structure grown consisted of two $5.5 \mathrm{~nm}$ wide GaAs QW doped with Si to $2 \times 10^{18} \mathrm{~cm}^{-3}$ separated by a $3 \mathrm{~nm} \mathrm{Al}_{0.25} \mathrm{Ga}_{0.75} \mathrm{As}$ barrier. This structure is sandwiched between two $\mathrm{Al}_{0.25} \mathrm{Ga}_{0.75} \mathrm{As}$ barriers $30 \mathrm{~nm}$ wide. The quality of the material regrown was evaluated by four different techniques: transmission electron microscopy (TEM), scanning electron microscopy (SEM), $\mathrm{X}$-ray diffractometry (XRD) and atomic force microscopy (AFM). Fig. 3 shows the results of the characterization, where it can be seen how the structural quality of the epilayer is comparable to the quality of the native substrate, both in surface roughness and crystal quality.

In the next step (e), we performed mesa etching to isolate the device. Then, two $100 \mu \mathrm{m} \times 100 \mu \mathrm{m}$ square wells were etched by RIE and filled with AuGe/Au (f) to fabricate the lateral ohmic contacts. Finally, the metallization of the Schottky bias and pinch-off gates

a

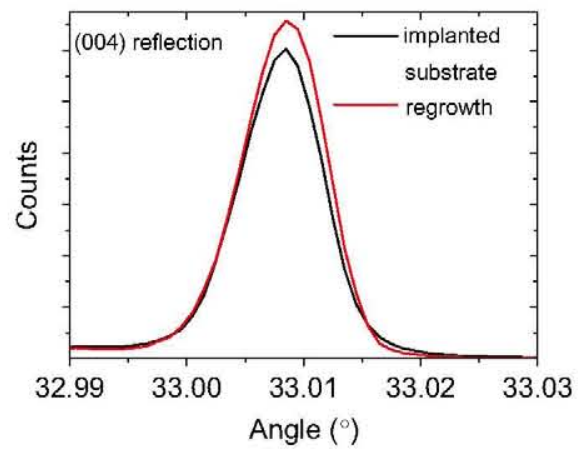

C

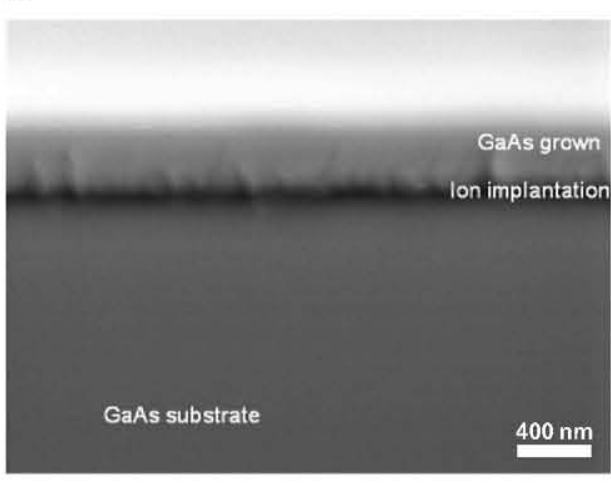

was performed using standard Joule evaporation of Au and further lift-off (g).

This final device was measured in an optical bench consisting of a glowbar, an IR monochromator and ZnSe lenses. The photosignal was collected by a lock-in amplifier.

\section{Results and discussion}

To assess the quality and performance of the processing procedure, we measured the $I-V$ characteristics of the device using a Hewlett-Packard 4145 parameter analyzer. Since the lateral contacts are $100 \mu \mathrm{m} \times 100 \mu \mathrm{m}$ square shaped, the thickness of the QWs is $5.5 \mathrm{~nm}$ and the length between contacts is $1 \mathrm{~mm}$, we can estimate the resistance of the QWs applying the following equation:

$R=\frac{1}{2} \frac{1}{q \mu_{\mathrm{e}}} n \frac{L}{d w}$

where $q$ is the electron charge, $\mu_{\mathrm{e}}$ is the electron mobility ( $3000 \mathrm{~cm}^{2} / \mathrm{V}$ s measured by Hall in bulk n-type GaAs samples), $n$ is the number of free carriers in the wells (equivalent to the doping level at room temperature $=2 \times 10^{18} \mathrm{~cm}^{-3}$ ), $L$ is the length between contacts, $d$ is the QW thickness and $w$ is the lateral dimension of the contact. This equation yields a result of $R=20 \mathrm{k} \Omega$, which is in good agreement with the $I-V$ characteristic shown in Fig. 4(a), where it can be observe a clear ohmic behaviour with a resistance of $14 \mathrm{k} \Omega$.

We also measured the $I-V$ curve of the pinch-off gate with respect to one of the ohmic contacts, which is chosen as the ground. Fig. 4(b) shows a good rectifying behaviour, with 4 orders of b

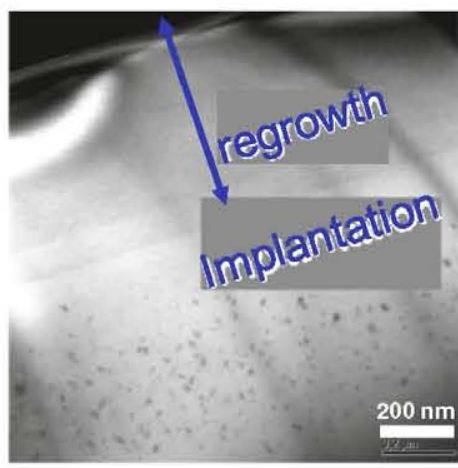

d

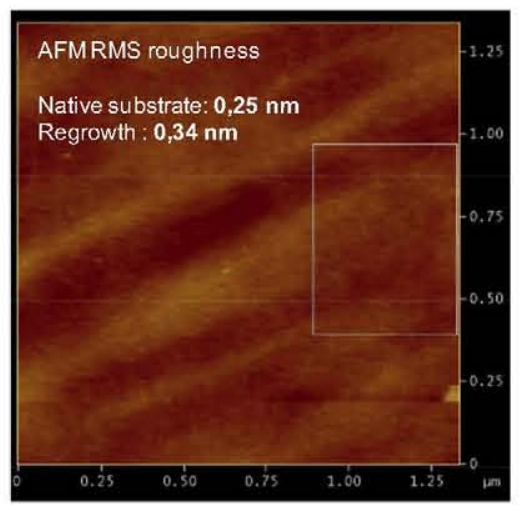

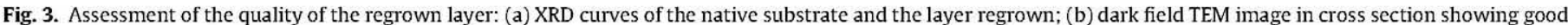
crystal quality; (c) SEM image showing the implanted and regrown layers and (d) AFM image showing good surface roughness in comparison with the substrate. 
a

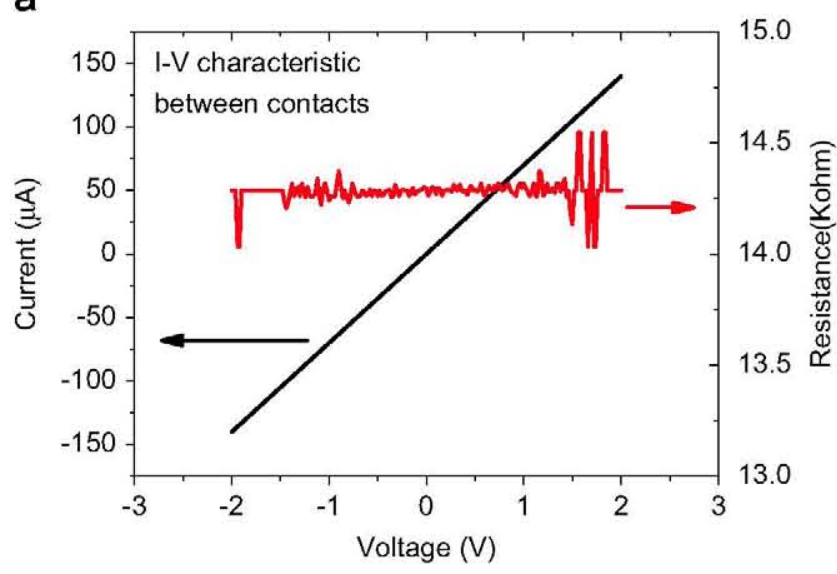

b

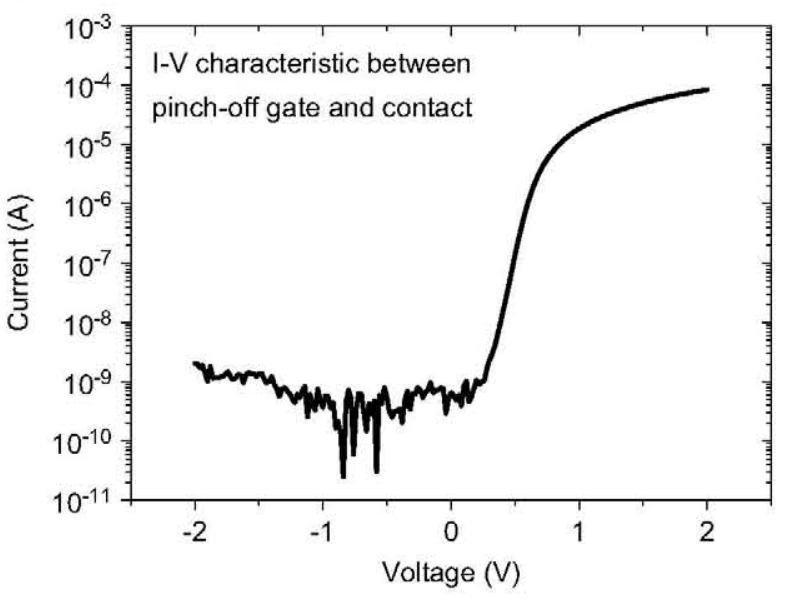

Fig. 4. I-V characteristic of (a) the laterally biased device measured between lateral contacts and (b) the Schottky pinch-off gate with respect to one of the lateral contacts.

magnitude of difference between the measured current in direct and reverse bias.

Since the normal incidence absorption is strongly reduced due to the selection rules for intersubband transitions in a quantum well, the QW device should show a very weak signal measured in those conditions. Therefore, we decided to test the device processing using a different sample with quantum dots (QD) in the active region (QDIP). The sample (shown in Fig. 5(a)) consists of 25 layers of InAs QD embedded in GaAs capping layers separated by $100 \mathrm{~nm}$. This structure is sandwiched between two $40 \mathrm{~nm}$ thick $\mathrm{Al}_{0.3} \mathrm{Ga}_{0.7} \mathrm{As}$ layers to block the dark current during the vertical transport. Finally, two Si doped $\left(2 \times 10^{18} \mathrm{~cm}^{-3}\right)$ GaAs layers are used as carrier collectors at both sides of the structure. The full epilayer was grown by MBE using the same chamber, although optimum growth conditions for the development of these nanostructures were employed. A piece of this QD sample was processed into conventional vertical photodetectors with $200 \mu \mathrm{m}$ diameters using standard photolithography and wet chemical mesa etching. A ring metallization using AuGe/Au was deposited on top of the mesa and then alloyed for the ohmic contacts. Another piece of the sample was processed in laterally biased detectors using the procedure mentioned above. The schematics of those processings are also detailed in Fig. 5(a).

In this sample the photocurrent mechanism is different; it is due to the intersubband transition between the QD states and the capping layer subbands. The excited electrons are transferred to those bands, leading to changes in the capping conductance [12]. a

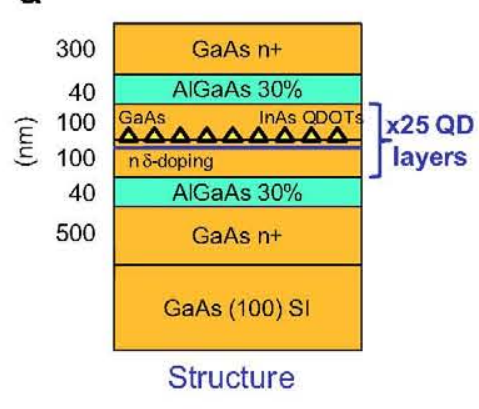

Vertical processing

Au / Ge ring

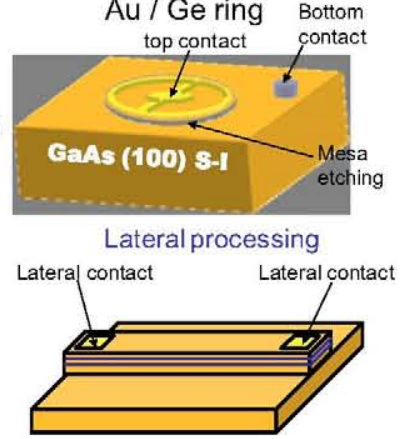

b

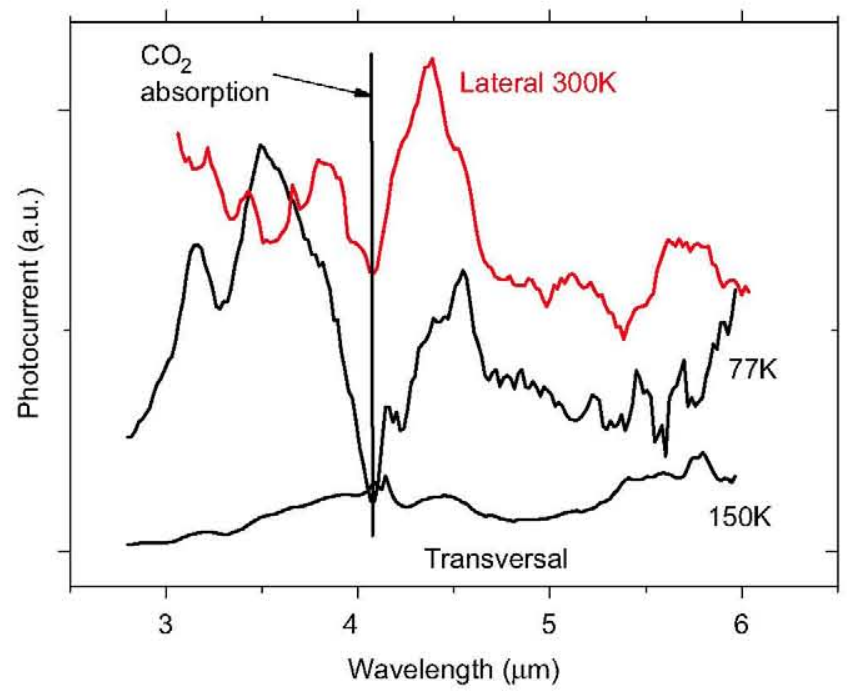

Fig. 5. (a) Structure of a QD infrared photodetector in conventional vertical and lateral configuration and (b) photoresponse of the device in both the configurations. The vertical structure allows operating up to $150 \mathrm{~K}$, the lateral structure increases the operating temperature to RT. As a reference, the peak value of the lateral structure corresponds to a responsivity of $0.6 \mathrm{~mA} / \mathrm{W}$.

We performed photocurrent measurements of the QD sample processed as conventional vertical photodetector at different temperatures using a closed cycle He cryostat. The bias voltage was $3 \mathrm{~V}$ with respect to the bottom contact, chosen as ground in all the measurements. In Fig. 5(b), we show the photosignals at 77 and $150 \mathrm{~K}$ (maximum practical operation temperature). We observed that the signal quenches above this temperature. This quenching can be attributed to the strong increment in the dark current from low temperatures $(15 \mathrm{~K}$ ) to $150 \mathrm{~K}$. At $150 \mathrm{~K}$, the difference in the intensity between the dark current and the photosignal is high enough to saturate the dynamic range of the lock-in amplifier. However, the lateral processing of the same sample allows increasing the operating temperature up to $300 \mathrm{~K}$, as it can also be seen in Fig. 5(b). In this configuration, it is possible to reduce the dark current by several orders of magnitude by means of the Schottky gates, thus providing a significant photocurrent signal even at high temperatures. As a reference, the peak value of the photocurrent signal corresponds to a responsivity of $0.6 \mathrm{~mA} / \mathrm{W}$.

Finally the photocurrent of the laterally biased QWIP was measured at normal incidence at room temperature, using a bias of $3.5 \mathrm{~V}$ between lateral contacts and $0 \mathrm{~V}$ in the Schottky gates. Fig. 6(a) shows a very clear peak centered at $8.5 \mu \mathrm{m}$, corresponding to the transition between the ground state and the first excited state in the QW. The sample absorption was previously measured by FTIR at a $45^{\circ}$ polished edge obtaining a ratio between $s$ and $p$ 


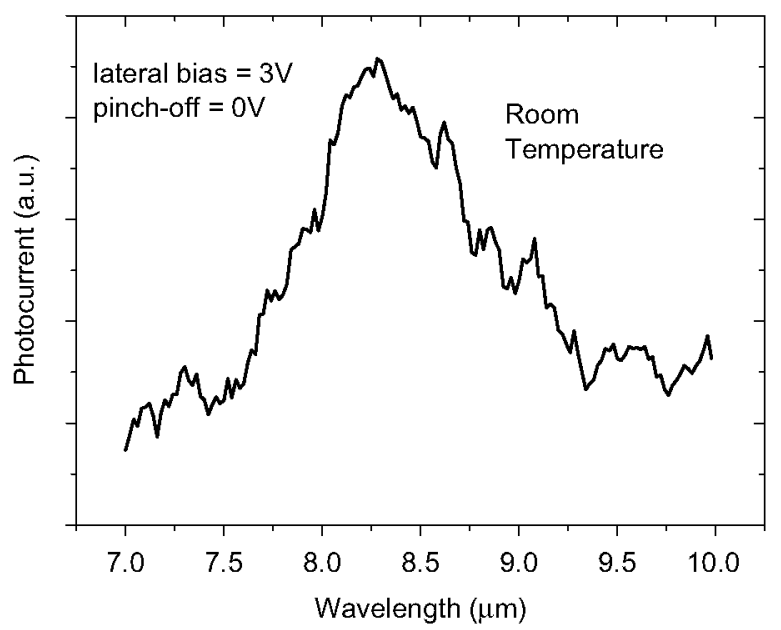

b

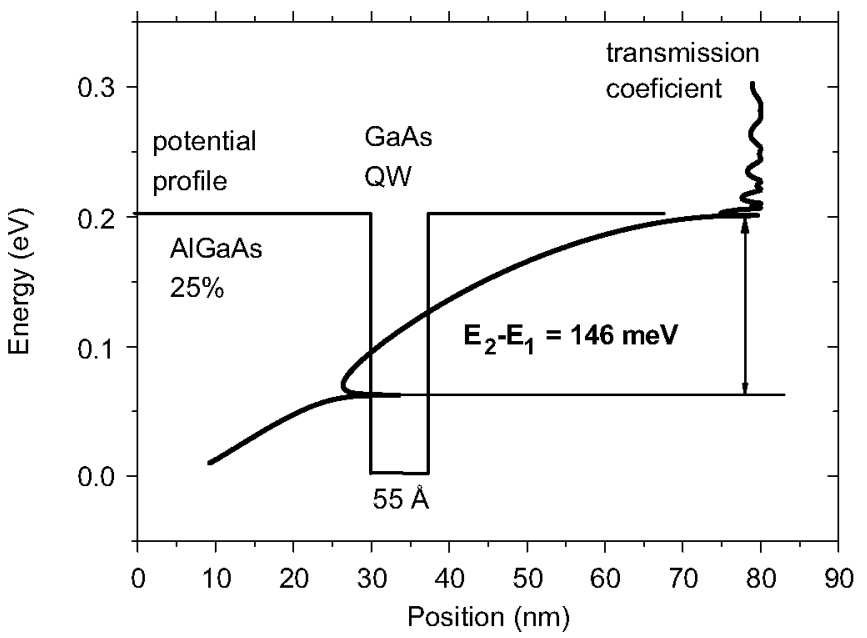

Fig. 6. (a) Room temperature photoresponse of a laterally biased QWIP measured with $3 \mathrm{~V}$ of lateral bias and $0 \mathrm{~V}$ in the Schottky pinch-off gates and (b) simulation by transfer matrix; the results agree with the experimental peak.

polarization, which demonstrates the intersubband character of the signal. Some authors have speculated about the possible origin of this significant photoresponse in normal incidence conditions, the diffraction of the light on the edges of the metal contacts and mesa etchings is one of the most likely explanations for this phenomenon [13]. An estimated value for the peak responsivity is $0.1 \mathrm{~mA} / \mathrm{W}$, and the noise in the dark current is calculated to be $2.8 \times 10^{-11} \mathrm{~A}_{\text {rms }}$. Taking into account the area of the device and the bandwidth of the noise measurement $(1 \mathrm{~Hz})$, the estimated $D^{*}$ is $1.07 \times 10^{5} \mathrm{~cm} \mathrm{~Hz}^{1 / 2} / \mathrm{W}$.

We simultaneously simulated the QW structure by means of a transfer matrix method to calculate the transmission coefficient using Airy functions. This method, predicts a difference of $146 \mathrm{meV}$ between the resonances corresponding to the ground level and the first confined state in a $5.5 \mathrm{~nm} \mathrm{QW}$, surrounded by $\mathrm{Al}_{0.25} \mathrm{Ga}_{0.75} \mathrm{As}$ barriers and kept under a bias voltage of $3 \mathrm{~V}$. This value is in good agreement with the peak measured at $8.5 \mu \mathrm{m}$ (equivalent to a difference of $145 \mathrm{meV}$ ). The results of this simulation are also included in Fig. 6(b).

\section{Conclusions}

In conclusion, we demonstrated the practical fabrication of laterally biased quantum infrared photodetectors with pinch-off Schottky gates to reduce the dark current due to direct conduction at room temperature. The devices were regrown by MBE on selective ion implanted substrates. The quality of the layer regrown is proved to be similar to the quality of the native substrate. The devices were tested using different characterization techniques, including $I-V$ characteristics and photocurrent spectroscopy. We observed clear photoresponse at room temperature in both QW and QD based detectors processed in lateral configuration in comparison with the conventional vertical processing, where it is not possible to separate the photosignal from the dark current. The results show a very good degree of agreement with the theory in all the cases.

\section{Acknowledgements}

The authors thank the European Office for Aerospace Research and Development (EOARD) for its sponsorship. The TEM image is courtesy of Dr. E. Luna, Paul Drude Institut für Festkörperelektronik, Hausvogteiplatz 5-7, 10117 Berlin (Germany).

\section{References}

[1] S.D. Gunapala, S.V. Bandara, J.K. Liu, et al., Demonstration of megapixel dualband QWIP focal plane array, IEEE Journal of Quantum Electronics 46 (2) (2010) 285-293.

[2] 〈http://www.irnova.se >, last access: August 2010

[3] T. Fujii, P. Masalkar, H. Nishino, et al., Comparison of performance between OWIPs with different two-dimensional random-grating optical couplers, Infrared Physics and Technology 42 (3-5) (2001) 199-204.

[4] B.F. Levine, Quantum well infrared photodetectors, Journal of Applied Physics 74 (8) (1993) R1-R81.

[5] M.B. El Mashade, M. Ashry, A. Nasr, Theoretical analysis of quantum dot infrared photodetectors, Semiconductor Science and Technology 18 (2003) $891-900$.

[6] Jong-Wook Kim, Jae-Eung Oh, Hong Seong-Chul, Chung-Hoon Park, TaeKyung Yoo, Room temperature far infrared $(8-10 \mu \mathrm{m})$ photodetectors using self-assembled InAs quantum dots with high detectivity, Applied IEEE Electron Device Letters 21 (7) (2000)

[7] P. Bhattacharya, X.H. Su, S. Chakrabarti, G. Ariyawansa, A.G.U. Perera, Characteristics of a tunneling quantum-dot infrared photodetector operating at room temperature, Applied Physics Letters 86 (2005) 191106.

[8] L. Chu, A. Zrenner, M. Bichler, G. Abstreiter, Quantum-dot infrared photodetector with lateral carrier transport, Applied Physics Letters 79 (14) (2001) 2249-2251.

[9] L. Chu, A. Zrenner, G. Böhm, G. Abstreiter, Lateral intersubband photocurrent spectroscopy on InAs/GaAs quantum dots, Applied Physics Letters 76 (14) (2000) 1944-1946.

[10] P.M. Alsing, D.A. Cardimona, D.H. Huang, T. Apostolova, W.R. Glass, C.D. Castillo, Advanced space-based detector research at the Air Force Research Laboratory, Infrared Physics and Technology 50 (2007) 89-94.

[11] A. Guzmán, J.L. Sánchez-Rojas, J.M.G. Tijero, J. Hernando, E. Calleja, E. Muñoz G. Vergara, R. Almazán, L.J. Gómez, M. Verdú, M.T. Montojo, Growth and characterization of a bound-to-quasi continuum QWIP with Al-graded triangular confinement barriers, IEEE Photonics Technology Letters 11 (12) (1999) $1650-1652$

[12] L. Chu, A. Zrenner, M. Bichler, G. Abstreiter, Quantum-dot infrared photodetector with lateral carrier transport, Applied Physics Letters 79 (14) (2001) 2249.

[13] H.C. Liu, M. Buchanan, Z.R. Wasilewski, M. Ramsteiner, D.S. Jiang, J.S. Harris, K.H. Ploog, How good is the polarization selection rule for intersubband transitions?, Applied Physics Letters 72 (14) (1998) 1682-1684. 